medical literature into a form easily assimilable by busy practitioners is defining and producing practice guidelines. Many practice guideline programmes exist, both in the United States (for example the NIH Consensus Conference program which has been running since 1977) and in various European countries. But there is little evidence that the diffusion of such guidelines effectively modifies clinical practice. ${ }^{17}$ As sociologist Ann Greer explains, mere diffusion of written matter is not sufficient to modify practices ingrained in behavioural, social, and local factors. ${ }^{18}$ Some of the complexities of implementing guidelines in practice are described by Richard Grol in this issue (p 184). ${ }^{19}$

An approach to resolving uncertainty of medical practice would be to develop and integrate three disciplines - medical decision analysis, clinical epidemiology, and evaluation of the quality of health care - into medicine and thus put scientific principle into everyday clinical practice. Medical decision analysis can be described as "a systematic approach to medical decision making under conditions of uncertainty." ${ }^{20}$ It is an aid to reasoning in that it separates the logical structure of a decision into its component parts and quantifies the probabilities of the different possible outcomes. The use of decision analysis forces the decision makers to consider explicitly the reasons and consequences of each choice. This allows each decision to be explained and justified to patients, to other health care workers and to the decision makers themselves.

Clinical epidemiology - the application of the principles and methods of epidemiology to clinical practice - promotes the practice of "a more systematic approach to gathering and interpreting clinical evidence" 21 and provides a framework for answering, with scientifically based argument, questions such as, "How accurate are the diagnostic tests or strategies I've applied? What will the consequences be of having this disease? How will the treatment I've prescribed change its future course and affect the patient?"22 Clinical epidemiology thus drives medical practice away from empiricism and tends to give it the characteristics of a science: it has been described as "the basic science of clinical practice." 21

Finally, through systematic evaluation of the quality of clinical care it is possible to assess the quality of the structure of care, the appropriateness and quality of medical procedures, and the desirability of outcomes for the individual patient. ${ }^{23}$ For this a methodical, rigorous, problem solving cycle which follows scientific principle is applied. ${ }^{24}$ By using an explicit and structured approach subjective influences are minimised and problems and their nature can be identified and prioritised; solutions and corrective measures may then be worked out and their implementation is verified to ensure their use and their effectiveness in producing the expected difference. ${ }^{25}$

Through acknowledging and understanding the presence of the variations and uncertainties in medical practice, practitioners may at least be able to give more open explanations of choices available to their patients. But application of the three disciplines could itself rationalise clinical practice and help diminish some of the uncertainty and variability in the practice of medicine - which must be in the best interest of individual patients. Integration of these disciplines into current clinical culture is likely to be slow. But many health professionals are now developing and using techniques for evaluating the quality of care. It is vital that all three disciplines should be integrated into medical and clinical studies so that clinical interventions can be used rationally and, when little evidence exists to support one approach or another, clinicians can inform patients to enable them to choose.

ALEXANDRA GIRAUD

Public Health Specialist,

Epidémiologie, Santé Publique,

Hôpital fean Verdier,

Assistance Publique Hôpitaux de Paris,

F-93143 Bondy Cedex,

France

1 Morin E. Science avec conscience. Paris: Fayard, 1983: 67

2 Bernard C. Introduction à la médicine expérimentale. Paris, 1865. Paris: Flammarion, Collection Champs, 1985.

3 Dubos R. Mirage of health. George Allen and Unwin, 1960.

4 Illich I. Némésis médicale. L'expropriation de la santé. Paris: Le Seuil, 1975.

5 McKeown T. The role of medicine. Oxford: Blackwell, 1979.

6 Jenicek M, Cléroux R. Epidémiologie clinique. Clinimétrie. Québec, Paris: Edisem Maloine, 1985.

7 Wennberg J, Gittelsohn. Variations in medical care among small areas. Sci Am 1982;246:120-34.

8 Chassin MR, Kosecoff J, Winslow CM. Does inappropriate use explain geographic variations in the use of medical services? A study of three procedures. $尹 A M A 1987 ; 258: 2533-7$.

Eddy $\mathrm{D}$. The challenge. $\mathscr{Y} A M A$ 1990;263:287-90.

10 De Solla Price D. The development and structure of the biomedical literature. In: Warren KS, ed. Coping with the biomedical literature. New York: Praeger, 1981.

11 Gremy F. Informatique médicale. Introduction à la méthodologie en médecine et santé publique. Paris: Flammarion, 1987.

12 Warren KS. Qualitative aspects of the biomedical literature. In: Warren $\mathrm{KS}$, ed. Coping with the biomedical literature. New York: Praeger, 1981.

13 Bernier L, Yerkey AN. Cogent communication: overcoming reading overload. Westport: Greenwood, 1979.

14 Williamson JW, Goldschmidt PG, Colton T. The quality of medical literature: an analysis of validation assessments. In: Bailar JC, Mosteller F, eds. Medical use of statistics. Waltham, Massachusetts: NEJM Books,

15 Wennberg JE. The paradox of appropriate care. $\mathcal{H} A M A$ 1987;258:2568-9.

16 Wennberg J. Better policy to promote the evaluative clinical sciences. Quality Assurance in Health Care 1990;1:21-9.

17 Giraud A, Jolly D. Les conférences de consensus ont-elles eu l'impact souhaité sur les pratiques médicales. In: Le consensus en médecine. Analyse et bilan des conférences de consensus dans le monde. Paris: AP-HP Doin, 1991:125-30.

18 Greer AL. The two cultures of biomedicine: can there be consensus? fAMA 1987;258:2739-40.

19 Grol R. Implementing guidelines in general practice. Quality in Health Care 1992;1:184-91.

20 Weinstein MC, Fineberg HV. Clinical decision analysis. Philadelphia: Saunders, 1980.

21 Sackett DL, Hayes RB, Tugwell P. Clinical epidemiology. A basic science for clinical medicine. Boston: Little, Brown, 1985.

22 Fletcher RH, Fletcher SW, Wagner EH. Clinical epidemiology. The essentials. 2nd ed. Baltimore: Williams and Wilkins, 1988.

23 Donabedian A. Explorations in quality assessment and monitoring. Vol 1. The definition of quality and approaches to its assessment. Ann Arbor, Michigan: Health Administration Press, 1985.

24 Russell IT, Wilson BJ. Audit: the third clinical science? Quality in Health Care 1992;1:51-5.

25 Giraud A. Evaluation medicale des soins hospitaliers. Paris: Economica, 1992

\title{
Measuring patient satisfaction for audit in general practice
}

Patient satisfaction surveys are currently being conducted by many family health services authorities (FHSAs) and medical audit advisory groups (MAAGs) in England and Wales. These are being used in an attempt to determine whether practices are providing a good standard of care as judged by their patients.
Unfortunately, the results are likely to be of only limited value because the validity of many of these questionnaires has not been tested and their sensitivity is often so restricted that most patients seem to express high levels of overall satisfaction.

General practitioners are also beginning to conduct 
patient satisfaction surveys to help them to improve the services they provide. Sometimes the surveys are conducted within the practice and sometimes in collaboration with neighbouring practices. The validity of these questionnaires is also often not established, and the design is undertaken by people with little experience of the methods. Some guidance is becoming available ${ }^{12}$; the most complete set of guidelines has been available for several years, in the form of standards for psychological tests agreed by the American Psychological Association. ${ }^{3}$ Two papers in this issue report the development and use of two questionnaires by a MAAG supported by an academic unit (p 153, 158). ${ }^{4}$ The authors emphasise the advantage of general practitioners and practice managers being involved in the development of the questionnaires, but any MAAGs which would like to follow this example and develop their own questionnaires would be wise to remember the commonly repeated advice to use already developed and tested examples whenever possible. The Newcastle upon Tyne questionnaires do show the value of some simple evaluation techniques such as the assessment of nonresponse to individual questions. Also a problem was demonstrated with conditional questions to be answered only by specific respondents, the other respondents being asked to move on to later questions, and this provides good evidence in support of the maxim to keep questionnaires simple.

The use of patient satisfaction surveys in health services research is well established. Such surveys are now being introduced into medical audit and this raises several new issues. Patients' views of their doctors include delicate personal information, and feedback will need to strike a balance between tact and providing clear guidance on what needs to be changed. Research on the most effective and useful forms of feedback is clearly required. Establishing the role of patient satisfaction surveys in encouraging change is also important, and evidence is required of the effects of these changes on subsequent patient satisfaction. The audit cycle must be completed. Do patients report improved satisfaction when appointment systems are revised to take their concerns into account? MAAGs must take extra care to ensure that the questionnaires they do use are adequately developed and documented. Providing information to practitioners which compares patients' opinions of their practice with those of other practices does place special demands on the questionnaires to be used. They must be sufficiently robust for the recipients of feedback to have confidence in the comparisons.

Encouraging change depends on the resolution of these issues. Eccles et al highlight the likelihood of involved practitioners instituting change ${ }^{5}$ whereas passive participants will often find reasons for ignoring the results of the survey. We do not know what happens to practices that simply receive unsolicited information about their performance, even if it is contrasted with the performance of neighbouring practices, although some evidence may become available from the experience of regular and intensive feedback about prescribing costs (PACT)

Improving the quality of care requires doctors and other health care workers to be involved in all stages of the audit cycle. Whether this should include the design of the audit tools themselves is presently unknown, but when this is practicable it may increase the likelihood of making appropriate changes within the practice when problems have been identified.

Because patient satisfaction is now seen as an important component of medical audit, many health professionals who are unfamiliar with questionnaire design will wish to undertake surveys. They should firmly resist the temptation to devise their own questionnnaires. Those researchers who do have the necessary skills should remember that robust tools are now required for medical audit. They should ensure that among those questionnaires that are developed are versions that can be used by inexperienced health professionals in a wide range of settings. Analysis must be simple and the findings must be presented in an easily understood format.

Patient opinion is far too important for it to be studied with inadequate measures. Poor questionnaires act as a form of censorship imposed on patients. They give misleading results, limit the opportunity of patients to express their concerns about different aspects of care, and can encourage professionals to believe that patients are satisfied when they are in reality highly discontented. In our enthusiasm to ask patients for their views we must avoid this disastrous trap. Through determined but sceptical inquiry and attention to detail there is a great deal that might be achieved. If reputable methods can be used and we take serious note of the findings, the therapeutic relationship between patients and their doctors can only improve.

Consultant Senior Lecturer

MICHAEL WHITFIELD

General Practice Unit,

Department of Epidemiology and Public Health Medicine,

University of Bristol,

Bristol BS8 2PR

Director,

RICHARD BAKER

Eli Lilly National Medical Audit Centre,

Department of General Practice,

University of Leicester School of Medicine,

Leicester General Hospital,

Leicester LE5 4PW

Fitzpatrick R. Surveys of patient satisfaction. I. Important general considerations. BMF 1991;302:887-9.

considerations. BMf $1991 ; 302: 887-9$.
Fitzpatrick R. Surveys of patient satisfaction. II. Designing a questionnaire and conducting a survey. $B M \mathcal{F}$ 1991;302:1129-32.

American Psychological Association. Committee to Develop Standards for Psychological Testing. Standards for educational and psychological testing. Washington, DC: APA, 1985.

4 Bamford C, Jacoby A. Development of patient satisfaction questionnaires. I. Methodological issues. Quality in Health Care 1992;1:153-7.

5 Eccles M, Jacoby A, Bamford C. Development of patient satisfaction questionnaires. II. Collaboration in practice. Quality in Health Care 1992;1:158-60. 